\title{
Correspondence
}

\section{The ATM-BID pathway plays a critical role in the DNA damage response by regulating mitochondria metabolism}

\author{
Cell Death and Differentiation (2016) 23, 182; doi:10.1038/cdd.2015.154; published online 27 November 2015
}

\section{Dear Editor,}

We are writing this correspondence as an attempt to resolve controversy in the field regarding the role of BID in the DNA damage response (DDR). BID is a well-established $\mathrm{BH}$-only BCL-2 family member that has a critical role in regulating mitochondrial apoptosis. ${ }^{1}$ Previously we have reported that DNA damage induces the phosphorylation of BID on serines 61 and 78 by the ataxia-telangiectasia mutated (ATM) kinase, and that this phosphorylation is important for cell cycle arrest and inhibition of apoptosis. ${ }^{2}$ In addition, another group reported similar results in response to replicative stress. ${ }^{3}$ Nevertheless, these findings were challenged by a third group, which demonstrated that in response to DNA damage $B I D^{-1}$ cells underwent cell cycle arrest and apoptosis in a manner indistinguishable from wild-type cells, arguing that BID is dispensable for the DDR. ${ }^{4}$ This study did not however touch upon the connection between BID and ATM. Thus, the role of BID in the DDR in the minds of many was not resolved.

In an endeavour to resolve these differences and advance our knowledge, we generated $B I D^{S 61 A S}$ S78A $\left(B I D^{A A}\right)$ knock-in mice, in which endogenous BID is no longer capable of being phosphorylated by ATM. We found that $B I D^{A A}$ mice are hypersensitive to whole-body irradiation and that this hypersensitivity was due to premature entry of haematopoietic stem cells (HSC) into active cell cycle and increased levels of HSC apoptosis. ${ }^{5}$ We also demonstrated that loss of BID phosphorylation was associated with accumulation of BID at the mitochondria and elevated levels of mitochondrial reactive oxygen species (ROS), suggesting that basal phosphorylation of BID is critical for balancing mitochondrial ROS, thus regulating HSC quiescence.

Interestingly, $B I D^{-/}$mice are not hypersensitive to DNA damage and show no abnormal HSC phenotypes in steady state or stress-induced haematopoiesis. ${ }^{5}$ This is most likely due to the fact that deletion of BID attenuates overproduction of mitochondrial ROS, maintaining normal basal ROS. Therefore, $B I D^{-/}$ mice are not expected to have the same phenotypes as $B I D^{A A}$ mice. The differential sensitivity between these two mouse models in response to DNA damage supports the notion and explains the initial alleged contradiction that the changes in baseline ROS level per se rather than BID itself dictate the sensitivity to DNA damage. Thus, the new $B I D^{A A}$ mouse model helped us shed new light upon this controversy and demonstrated that BID does have a critical role in the DDR in vivo.
Our more recent findings suggest that BID regulates mitochondrial ROS/metabolism via mitochondrial carrier homolog 2 (MTCH2). MTCH2 acts as a mitochondrial receptor for BID essential for apoptosis, ${ }^{6}$ and is suspected to have a role in metabolism. ${ }^{7}$ Importantly, we recently found that loss of MTCH2 in the haematopoietic system results in premature entry of HSCs into cycle accompanied by a moderate increase in mitochondrial $\mathrm{ROS}^{8}{ }^{8}$ findings that were similar to the ones obtained with the $B I D^{A A}$ mice. Moreover, loss of MTCH2 or loss of BID phosphorylation $\left(B I D^{A A}\right)$ led to a substantial increase in multiple diverse mitochondrial metabolism parameters. Interestingly, loss of $\mathrm{MTCH} 2$ led to a protection from irradiation-induced apoptosis, ${ }^{8}$ whereas loss of BID phosphorylation sensitized cells to DNA damage-induced apoptosis, ${ }^{5}$ suggesting that changes in mitochondrial metabolism can either protect from or sensitize to apoptosis, probably depending on the ROS levels.

All these findings are consistent with the idea that BID serves as an upstream negative regulator of $\mathrm{MTCH} 2$ and that MTCH2 acts as a repressor of mitochondrial metabolism (Supplementary Figure S1). Hence, the ATM-BID-MTCH2 pathway that we have identified plays a critical role in the DDR via regulation of mitochondrial metabolism.

\section{Conflict of Interest}

The authors declare no conflict of interest.

\section{A Gross ${ }^{*, 1}$, Y Zaltsman ${ }^{1}$ and M Maryanovich ${ }^{2,3}$}

1 Department of Biological Regulation, Weizmann Institute, Rehovot, Israel;

2 Ruth L. and David S. Gottesman Institute for Stem Cell and Regenerative Medicine Research, Albert Einstein College of Medicine, Bronx, NY, USA and

${ }^{3}$ Department of Cell Biology, Albert Einstein College of Medicine, Bronx, NY, USA

* Corresponding author: A Gross, Department of Biological Regulation, Weizmann Institute of Science, PO BOX 26, Rehovot 76100, Israel. Tel: +972 8 9343656; Fax: +972 8 9344116; E-mail: atan.gross@ @eizmann.ac.il

\footnotetext{
1. Yin XM et al. Nature 1999; $400: 886-891$.

2. Kamer I et al. Cell 2005; 122: 593-603.

3. Zinkel SS et al. Cell 2005; 122: 579-591.

4. Kaufmann T et al. Cell 2007; 129: 423-433.

5. Maryanovich M et al. Nat Cell Biol 2012; 14: 535-541.

6. Zaltsman Y et al. Nat Cell Biol 2010; 12: 553-562.

7. Willer CJ et al. Nat Genet 2009; 41: 25-34.

8. Maryanovich M et al. Nat Commun 2015; 6: 7901.
} 